\title{
Pituitary Tumor-Transforming Gene 1 Is Expressed in Primary Ductal Breast Carcinoma, Lymph Node Infiltration, and Distant Metastases
}

\author{
Fabio Grizzi, ${ }^{1}$ Sonia Di Biccari, ${ }^{1}$ Barbara Fiamengo, ${ }^{1}$ \\ Sanja Štifter, ${ }^{2}$ and Piergiuseppe Colombo ${ }^{1}$ \\ ${ }^{1}$ Humanitas Clinical and Research Center, Via Manzoni 56, 20089 Rozzano, Milan, Italy \\ ${ }^{2}$ Department of Pathology, School of Medicine, University of Rijeka, Brace Branchetta St. No. 20, HR-51 000 Rijeka, Croatia
}

Correspondence should be addressed to Fabio Grizzi; fabio.grizzi@humanitasresearch.it

Received 18 June 2013; Accepted 28 August 2013

Academic Editor: Ferdinando Mannello

Copyright (C) 2013 Fabio Grizzi et al. This is an open access article distributed under the Creative Commons Attribution License, which permits unrestricted use, distribution, and reproduction in any medium, provided the original work is properly cited.

\begin{abstract}
Despite the advances that have been made in the fields of molecular and cell biology, there is still considerable debate explaining how the breast cancer cells progress through carcinogenesis and acquire their metastatic ability. The lack of preventive methods and effective therapies underlines the pressing need to identify new biomarkers that can aid early diagnosis and may be targets for effective therapeutic strategies. In this study we explore the pituitary tumor-transforming gene 1 (PTTG1) expression in primary ductal breast carcinoma, lymph node infiltration, and distant metastases. Three human cell lines, 184B5 derived from normal mammary epithelium, HCC70 from a primary ductal carcinoma, and MDA-MB-361 from a breast metastasis, were used for quantifying PTTG1 mRNA expression. The PTTG1 immunohistochemical expression was carried out on specimens taken from eight patients with invasive ductal breast cancer who underwent surgical treatment and followup for five years retrospectively selected. The study demonstrated that PTTG1 is expressed gradually in primary ductal breast carcinoma, lymph node infiltration, and distant metastases. Our findings suggest that the immunohistochemical evaluation of PTTG1 expression might be a powerful biomarker of recognition and quantification of the breast cancer cells in routine pathological specimens and a potential target for developing an effective immunotherapeutic strategy for primary and metastatic breast cancer.
\end{abstract}

\section{Introduction}

Breast cancer is by far the most frequent cancer in women (23\% of all cancers) and the second-most frequent cancer when both sexes are considered [1]. Incidence rates are high in the most of the developed world (except for Japan, where it comes third after colorectal and stomach cancer), the highest age-standardized incidence being observed in North America, where it is the most common malignant disease [2].

Historically, breast cancer was percept as a linear multistep process involving progressive changes from normal to hyperplastic with or without atypia, carcinoma in situ, invasive carcinoma, and local and distant metastases, but it is now agreed that it is a highly heterogeneous disease encompassing a wide variety of pathological entities and a range of clinical behaviors [3-6]. This acquired general statement is underpinned at molecular level by a complex array of genetic alterations that affect cell processes and determine the self-progression of the neoplastic disease and its response to therapy $[6,7]$.

Pituitary tumor-transforming gene 1 (PTTG1) is a wellknown homologue of the yeast securin, which arrests the activation of separin and induces sister chromatid separation in the transition from metaphase to anaphase [8]. Originally cloned from rat pituitary tumor cells [8], it has been found over-expressed in a variety of human endocrine-related neoplasms (especially pituitary, thyroid, ovarian, and uterine tumors) and other cancers involving the central nervous, respiratory, and gastrointestinal systems $[9,10]$. It is known that the over-expression of PTTG1 leads to aneuploidy, causes in vitro transformation and tumor formation in vivo, regulates basic-fibroblast growth factor secretion, and induces 


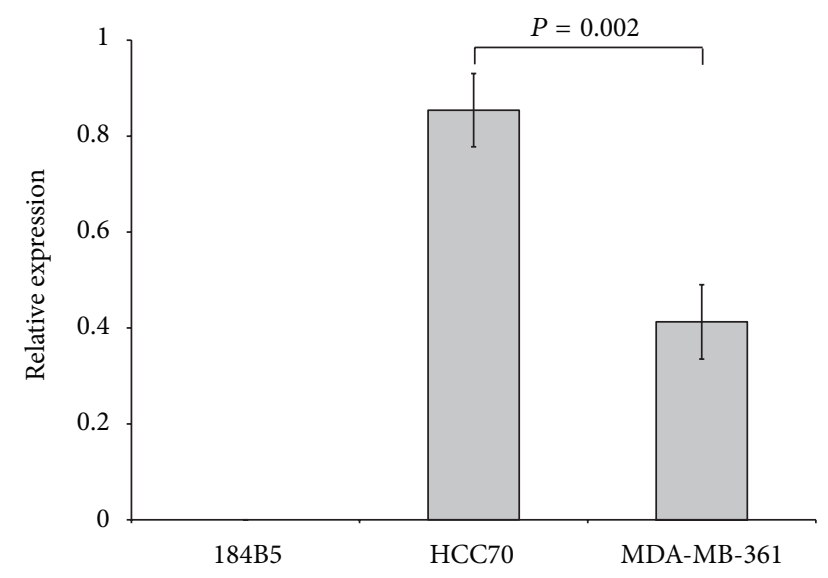

FIGURE 1: Quantification of PTTG1 transcript levels in normal breast epithelium cell line 184B5, primary breast tumor cell line HCC70, and in a breast metastatic cell line MDA-MB-361, by qRT-PCR. PTTG1 mRNA was higher in HCC70 and MDA-MB-361 than in the 184B5 cell line and higher in HCC70 than in the MDA-MB-361 cell line $(P=0.002)$.

angiogenesis [11]. Furthermore, the level of mRNA expression encoding PTTG1 has been mainly associated with tumor aggressiveness [11-20]. We here demonstrate that PTTG1 is expressed in primary ductal breast cancer, infiltrated lymph node, and distant metastases, and we briefly discuss its potential to help in defining effective immunotherapeutic strategies for primary and secondary breast cancer.

\section{Materials and Methods}

2.1. Cell Lines. Three human cell lines purchased from the American Type Culture Collection (ATCC, Manassas, USA), 184B5 derived from normal mammary epithelium, HCC70 from a primary ductal carcinoma, and MDA-MB-361 from a breast metastasis, were used for quantifying PTTG1 mRNA expression. HCC70 cells were cultured in RPMI 1640 medium (Sigma, Milan, Italy) supplemented with $2 \mathrm{mM} \mathrm{L}$ glutamine, $10 \mathrm{mM}$ HEPES, $1 \mathrm{mM}$ sodium pyruvate, $4.5 \mathrm{~g} / \mathrm{L}$ glucose, $1.5 \mathrm{~g} / \mathrm{L}$ sodium bicarbonate, and $10 \%$ fetal bovine serum (FBS, Sigma). MDA-MB-361 cells were cultured in Leibovitz's L-15 medium (Sigma) supplemented with 20\% FBS, and 184B5 cells were cultured in serum-free Mammary Epithelial Growth Medium (MEGM, Cambrex BioScience, Milan, Italy) supplemented with $1 \mathrm{ng} / \mathrm{mL}$ cholera toxin (Sigma). All the cells were maintained at $37^{\circ} \mathrm{C}$ in a humidified $5 \% \mathrm{CO}_{2}$ atmosphere.

2.2. Patients. Eight patients with invasive ductal breast cancer who underwent surgical treatment and followup for five years at the Humanitas Clinical and Research Center, Rozzano, Milan, Italy, were retrospectively selected (Table 1). For each patient histological grade, pathological stage, estrogen and progesterone receptors status, HER-2/new, MIB-1, and the number of positive lymph nodes were known.
2.3. Real-Time Quantitative Reverse-Polymerase Chain Reaction. PTTG1 mRNA was quantified in 184B5, HCC70, and MDA-MB-361 cell lines by means of a standardized real-time quantitative RT-PCR (qRT-PCR, Celbio, Foster City, CA, USA). $\Delta \Delta$ Ct was used to calculate transcript levels relative to $\beta$-actin (Applied Biosystems, Milan, Italy). qRT-PCR was performed at least three times.

2.4. Immunohistochemistry. For the immunohistochemistry $2 \mu \mathrm{m}$-thick sections of each surgical specimen were cut and processed. After deparaffining and rehydration, they were immersed in an antigen retrieval bath (Dako, Milan, Italy), incubated with $3 \% \mathrm{H}_{2} \mathrm{O}_{2}$ for $15 \mathrm{~min}$ to quench endogenous peroxidase activity, treated for two hours at room temperature with primary antibodies raised against PTTG1 (Zymed, South San Francisco, CA, USA) or with rabbit IgG (Dako) as a negative control, and then incubated for 30 minutes with the DAKO Envision system (Dako). 3,3'diaminobenzidine tetrahydrochloride (Dako) was used as a chromogen to yield brown reaction products. The nuclei were lightly counterstained with hematoxylin solution (Diapath, Bergamo, Italy).

2.5. Statistical Analysis. All of the data are expressed as mean values \pm standard deviation. The results were analyzed using Statistica software (StatSoft Inc., Tulsa, OK, USA). Univariate analysis was performed by means of the Student's $t$ test as required for parametric variables. A $P$ value of less than 0.05 was considered to be statistically significant.

\section{Results and Discussion}

We found that the expression of mRNA encoding PTTG1 was higher in HCC70 and MDA-MB-361 than in the 184B5 cell line and higher in HCC70 than in the MDA-MB-361 cell line $(0.854 \pm 0.076$ versus $0.412 \pm 0.077 ; P=0.0021)$ (Figure 1$)$.

Furthermore, immunostaining of PTTG1 demonstrated that it is highly expressed in primary breast cancers and homogeneously distributed in tumoral cells disseminated in lymph nodes and colonizing distant anatomical sites (Figure 2).

These findings extend previous observations showing that, in comparison with normal breast ductal epithelium, PTTG1 mRNA is overexpressed in primary and metastatic breast cancer cell lines [18, 21, 22] and first define PTTG1 as an antigen expressed in the breast cancer cells making up the primary tumoral mass, the tumoral cells infiltrating the lymph nodes, and those metastasizing to distant organs.

Despite the significant advances in diagnosing and treatment of breast cancer, a number of unresolved clinical questions still remain. They regard the prevention, diagnosis, tumor progression and recurrence, treatment, and therapeutic resistance [23]. Resolving all these problems is complicated by the fact that breast cancer is not a single disease but is highly heterogeneous at both the molecular and clinical level $[3,5,23]$.

Although several studies of gene expression have highlighted expression profiles and gene sets that are prognostic, 


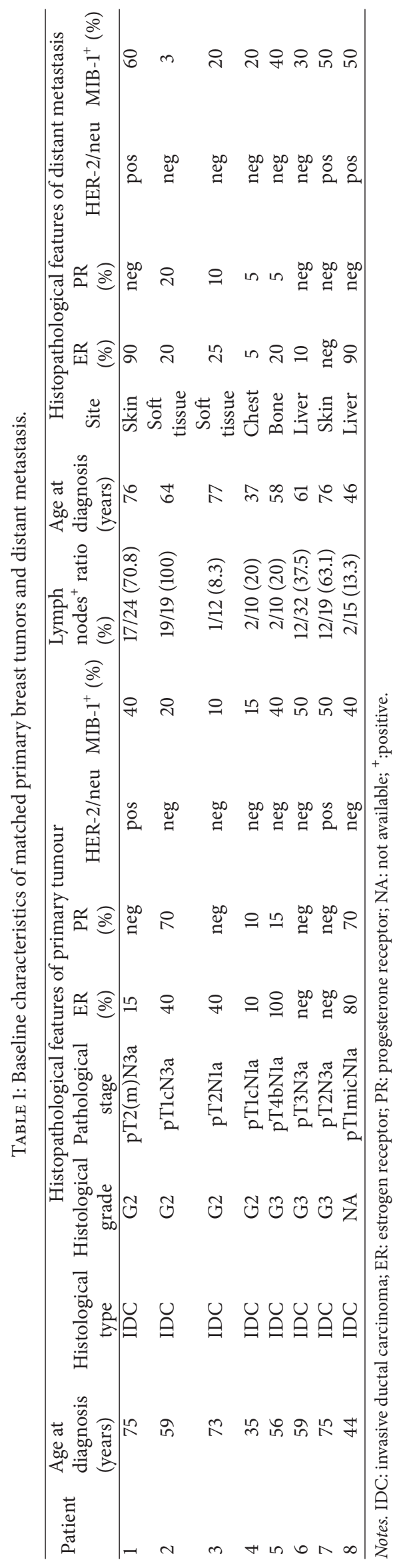


Distant metastasis

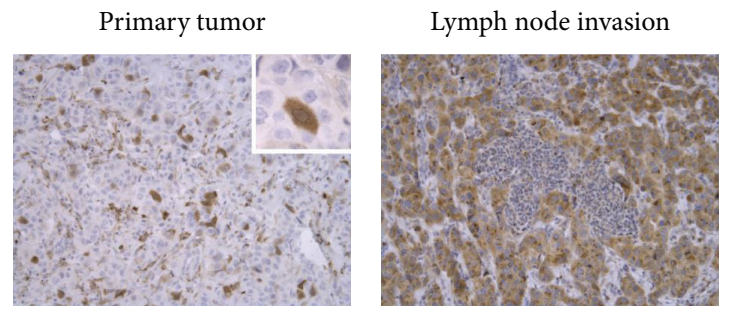

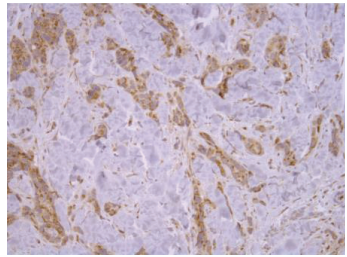

(a)

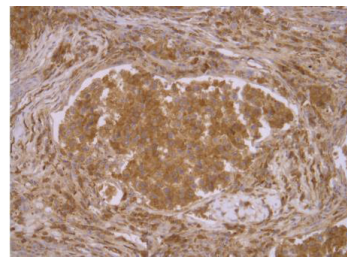

(c)

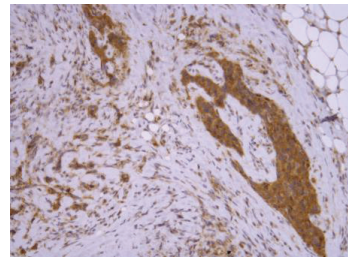

(e)

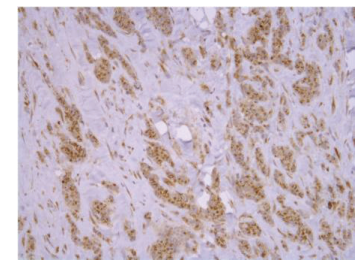

(b)

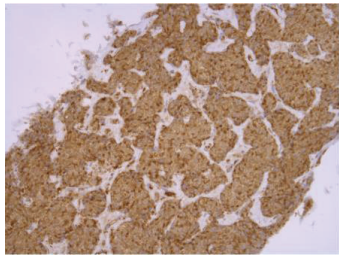

(d)

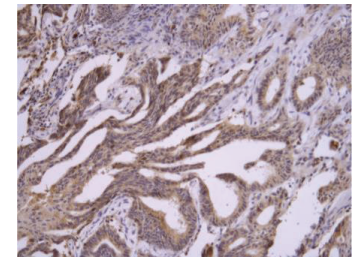

(f)

FIGURE 2: PTTG1 is an antigen constitutively expressed in the breast cancer cells making up the primary tumoral mass, the tumoral cells invading the lymph node, and those metastasising to distant organs including skin (a), soft tissues (b and c), liver (d), bone (e), and chest (f). Objective magnification 20x (inset 63x).

predictive, or both for patients with breast cancer [24, 25], conventional prognostic factors, such as TNM stage and certain histopathological features (i.e. grade, lymphovascular invasion, estrogen and progesterone receptor status, and human epidermal growth factor receptor 2), still broadly categorize patients into low-, intermediate-, and high-risk groups. However, even so, if these help in treatment planning, it has been demonstrated that such conventional information are really insufficient and that there is a need for additional histopathological markers [3, 25-27]. Furthermore, there is growing evidence that the detection of occult lymph node micrometastases not revealed by a standard pathological examination but with sentinel lymph node biopsy can identify patients at increased risk of recurrence and reduced survival.

The PTTG1 protooncogene has been detected in several tumors of unrelated histological origin, and its level of expression has been associated with the aggressiveness of the primary neoplasia $[8,11]$. The present study firstly demonstrates that it can be entitled as a useful histopathological marker of breast carcinogenesis progression. The expression pattern of PTTG1 in primary and metastatic breast cancer cells and its restricted expression in normal tissues suggest it to be an important molecular target in the search for new antigens and immunotherapeutic strategies for breast cancer. Since in the breast cancer the PTTG1 is showing opposite expression pattern of pathway analogues such as securin [28], its detection could be of great clinical importance, being informative for the tumor proliferative capacity and very likely the simultaneous presence of the p53 oncogene mutation pointing towards worse prognosis, shorter time to relapse, and disease progression. In our group only one patient had low proliferation index being insufficient for the speculation, but the immunoreactivity in the metastatic cells allows us to state the presence of the evidence regarding the coexistence of PTTG1 and more aggressive tumor phenotypes.

It has been shown that the genesis and progression of prostate cancer have mainly focused on the activation and expression of oncogenes and that PTTG1 is overexpressed in human prostate cancer tissue [29] which supports our findings. Huang et al. strongly suggest that PTTG1 is a critical mediator of prostate tumor growth and invasion [30]. Ishitsuka et al. have, recently, observed that the PTTG1 is a ubiquitously expressed transcription factor that can promote an enhanced proliferation of cultured epidermal keratinocytes and that its expression correlates with proliferation, differentiation capacity, or the existence of mutated tumor suppressor genes [31]. Using small interfering RNA (siRNA) to silence PTTG1 expression in human A2780 ovarian carcinoma cells, El-Naggar et al. [32] assessed the effect of PTTG1 silencing on tumor formation in vitro and in vivo. The siRNA reduced the expression of both PTTG1 mRNA and protein, and a $50 \%$ reduction in cell proliferation was achieved in the cells constitutively expressing PTTG1 siRNA in comparison with vector or control siRNA-transfected cells. Furthermore, they showed that nude mice injected with A2780 cells constitutively expressing PTTG1-siRNA 
decreased the incidence of tumor development and growth [23]. It has also been found that using the siRNA to silence the PTTG1 gene is an effective method of treating liver cancer [33].

The paucity of target antigens is one of the major factors limiting the development of effective immunotherapeutic strategies. Moreover, it has been shown that a prerequisite for the success of any tumor-specific therapeutic strategy is the existence and identification of antigen targets that are not only exclusively or preferentially expressed in malignant tissues, but also expressed in a high percentage of neoplastic cells in a large number of patients. The present study first demonstrates that the immunohistochemical evaluation of PTTG1 expression is a potentially powerful mean of determining and quantifying breast cancer cells in routine pathological diagnosis. Further clinical studies are necessary to validate the observed potential of PTTG1 use as an antigen target for developing effective therapeutic strategies for primary and secondary breast cancer.

\section{References}

[1] R. Siegel, D. Naishadham, and A. Jemal, "Cancer statistics, 2012," CA: A Cancer Journal for Clinicians, vol. 62, no. 1, pp. 10-29, 2012.

[2] G. Arpino, R. Laucirica, and R. M. Elledge, "Premalignant and in situ breast disease: biology and clinical implications," Annals of Internal Medicine, vol. 143, no. 6, pp. 446-457, 2005.

[3] Cancer Genome Atlas Network, "Comprehensive molecular portraits of human breast tumours," Nature, vol. 490, no. 7418, pp. 61-70, 2012.

[4] A. Prat and C. M. Perou, "Deconstructing the molecular portraits of breast cancer," Molecular Oncology, vol. 5, no. 1, pp. 5-23, 2011.

[5] K. Polyak, "Is breast tumor progression really linear?" Clinical Cancer Research, vol. 14, no. 2, pp. 339-341, 2008.

[6] D. Colak, A. Nofal, A. Albakheet et al., "Age-specific gene expression signatures for breast tumors and cross-species conserved potential cancer progression markers in young women," PloS One, vol. 8, no. 5, Article ID e63204, 2013.

[7] M. Shipitsin, L. L. Campbell, P. Argani et al., "Molecular definition of breast tumor heterogeneity," Cancer Cell, vol. 11, no. 3, pp. 259-273, 2007.

[8] L. Pei and S. Melmed, "Isolation and characterization of a pituitary tumor-transforming gene (PTTG)," Molecular Endocrinology, vol. 11, no. 4, pp. 433-441, 1997.

[9] H. Zou, T. J. McGarry, T. Bernal, and M. W. Kirschner, "Identification of a vertebrate sister-chromatid separation inhibitor involved in transformation and tumorigenesis," Science, vol. 285, no. 5426, pp. 418-421, 1999.

[10] V. E. Smith, J. A. Franklyn, and C. J. McCabe, "Pituitary tumortransforming gene and its binding factor in endocrine cancer," Expert Reviews in Molecular Medicine, vol. 12, article e38, 2010.

[11] G. Vlotides, T. Eigler, and S. Melmed, "Pituitary tumortransforming gene: physiology and implications for tumorigenesis," Endocrine Reviews, vol. 28, no. 2, pp. 165-186, 2007.

[12] C. Sáez, M. A. Japón, F. Ramos-Morales et al., "hpttg is overexpressed in pituitary adenomas and other primarp epithelial neoplasias," Oncogene, vol. 18, no. 39, pp. 5473-5476, 1999.
[13] Y. Shibata, N. Haruki, Y. Kuwabara et al., "Expression of PTTG (Pituitary Tumor Transforming Gene) in esophageal cancer," Japanese Journal of Clinical Oncology, vol. 32, no. 7, pp. 233-237, 2002.

[14] C. Sáez, T. Pereda, J. J. Borrero et al., "Expression of hpttg protooncogene in lymphoid neoplasias," Oncogene, vol. 21, no. 53, pp. 8173-8177, 2002.

[15] S. Honda, M. Hayashi, Y. Kobayashi, Y. Ishikawa, K. Nakagawa, and E. Tsuchiya, "A role for the pituitary tumor-transforming gene in the genesis and progression of non-small cell lung carcinomas," Anticancer Research, vol. 23, no. 5A, pp. 3775-3782, 2003.

[16] A. P. Heaney, R. Singson, C. J. McCabe, V. Nelson, M. Nakashima, and S. Melmed, "Expression of pituitary-tumour transforming gene in colorectal tumours," The Lancet, vol. 355, no. 9205, pp. 716-719, 2000.

[17] C.-Y. Wen, T. Nakayama, A.-P. Wang et al., "Expression of pituitary tumor transforming gene in human gastric carcinoma," World Journal of Gastroenterology, vol. 10, no. 4, pp. 481-483, 2004.

[18] C. Solbach, M. Roller, C. Fellbaum, M. Nicoletti, and M. Kaufmann, "PTTG mRNA expression in primary breast cancer: a prognostic marker for lymph node invasion and tumor recurrence," Breast, vol. 13, no. 1, pp. 80-81, 2004.

[19] F. Salehi, B. W. Scheithauer, S. Sharma et al., "Immunohistochemical expression of PTTG in brain tumors," Anticancer Research, vol. 33, no. 1, pp. 119-122, 2013.

[20] F. Grizzi, B. Franceschini, S. Musardo, E. E. Frezza, E. Cobos, and M. Chiriva-Internati, "Pituitary tumor transforming gene 1 in the liver," Hepatology, vol. 44, no. 6, pp. 1701-1702, 2006.

[21] S. Ogbagabriel, M. Fernando, F. M. Waldman, S. Bose, and A. P. Heaney, "Securin is overexpressed in breast cancer," Modern Pathology, vol. 18, no. 7, pp. 985-990, 2005.

[22] C. H. Yoon, M. J. Kim, H. Lee et al., "PTTG1 oncogene promotes tumor malignancy via epithelial to mesenchymal transition and expansion of cancer stem cell population," The Journal of Biological Chemistry, vol. 287, no. 23, pp. 19516-19527, 2012.

[23] K. Polyak, "Breast cancer: origins and evolution," The Journal of Clinical Investigation, vol. 117, no. 11, pp. 3155-3163, 2007.

[24] C. Fan, D. S. Oh, L. Wessels et al., "Concordance among geneexpression-based predictors for breast cancer," The New England Journal of Medicine, vol. 355, no. 6, pp. 560-569, 2006.

[25] E. A. Rakha, M. E. El-Sayed, J. S. Reis-Filho, and I. O. Ellis, "Expression profiling technology: its contribution to our understanding of breast cancer," Histopathology, vol. 52, no. 1, pp. 67-81, 2008.

[26] B. Weigelt, J. L. Peterse, and L. J. van't Veer, "Breast cancer metastasis: markers and models," Nature Reviews Cancer, vol. 5, no. 8, pp. 591-602, 2005.

[27] V. V. Levenson, "Biomarkers for early detection of breast cancer: what, when, and where?" Biochimica et Biophysica Acta, vol. 1770, no. 6, pp. 847-856, 2007.

[28] K. Talvinen, J. Tuikkala, O. Nevalainen et al., "Proliferation marker securin identifies favourable outcome in invasive ductal breast cancer," British Journal of Cancer, vol. 99, no. 2, pp. 335340, 2008.

[29] X. Zhu, Z. Mao, Y. Na, Y. Guo, X. Wang, and D. Xin, "Significance of pituitary tumor transforming gene 1 (PTTG1) in prostate cancer," Anticancer Research, vol. 26, no. 2A, pp. 12531259, 2006. 
[30] S. Q. Huang, Q. J. Liao, X. W. Wang et al., "RNAi-mediated knockdown of pituitary tumor- transforming gene-1 (PTTG1) suppresses the proliferation and invasive potential of PC3 human prostate cancer cells," Brazilian Journal of Medical and Biological Research, vol. 45, no. 11, pp. 995-1001, 2012.

[31] Y. Ishitsuka, Y. Kawachi, S. Taguchi et al., "Pituitary tumortransforming gene 1 as a proliferation marker lacking prognostic value in cutaneous squamous cell carcinoma," Experimental Dermatology, vol. 22, no. 5, pp. 318-322, 2013.

[32] S. M. El-Naggar, M. T. Malik, and S. S. Kakar, "Small interfering RNA against PTTG: a novel therapy for ovarian cancer," International Journal of Oncology, vol. 31, no. 1, pp. 137-143, 2007.

[33] C.-R. Jung, J. Yoo, J. J. Ye et al., "Adenovirus-mediated transfer of siRNA against PTTG1 inhibits liver cancer cell growth in vitro and in vivo," Hepatology, vol. 43, no. 5, pp. 1042-1052, 2006. 


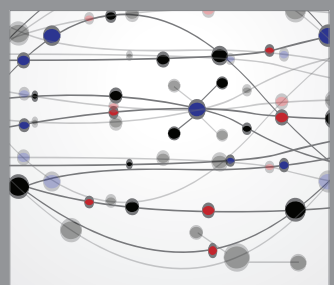

The Scientific World Journal
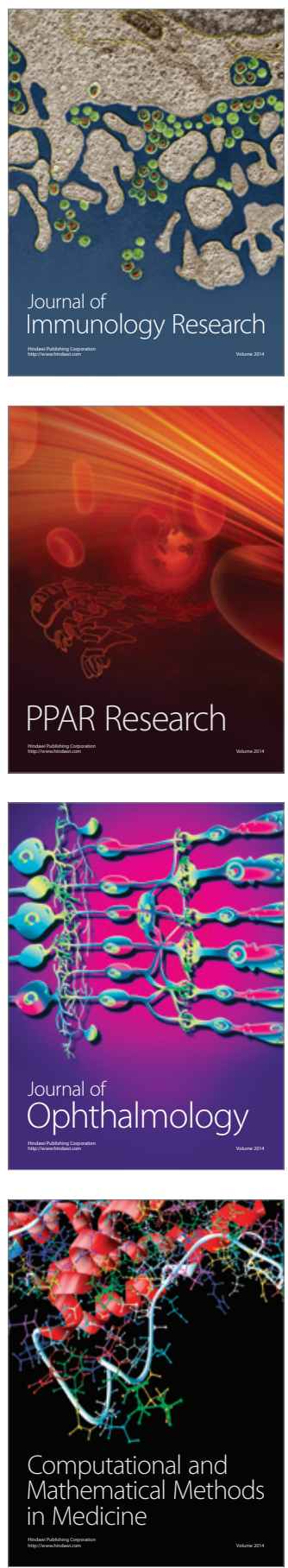

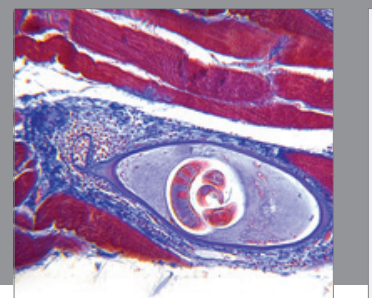

Gastroenterology

Research and Practice
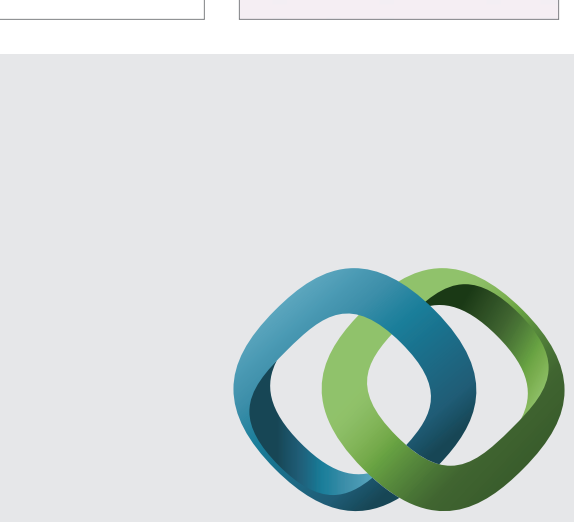

\section{Hindawi}

Submit your manuscripts at

http://www.hindawi.com
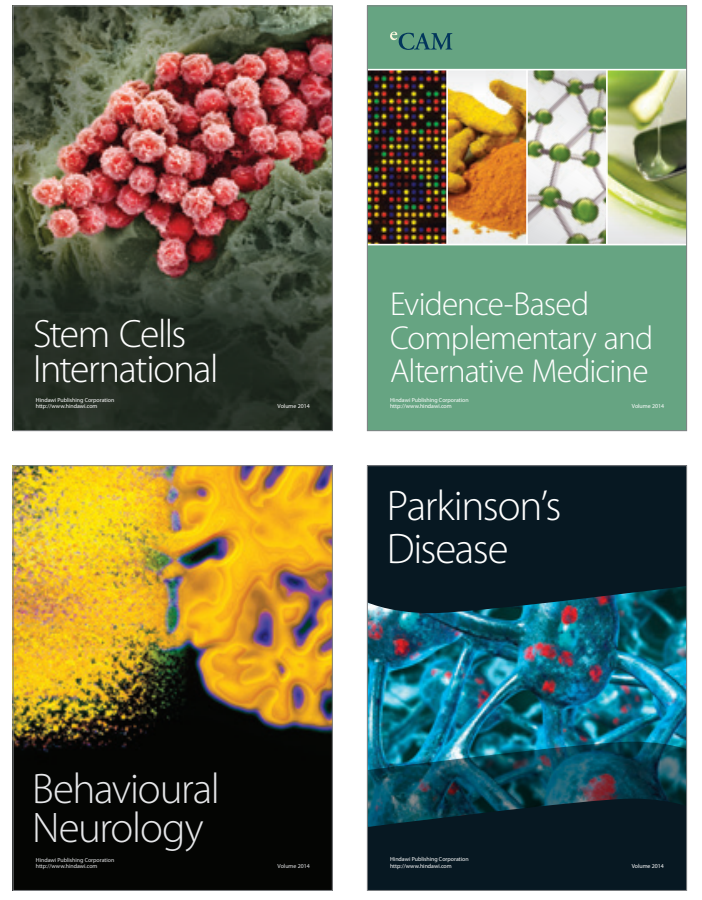
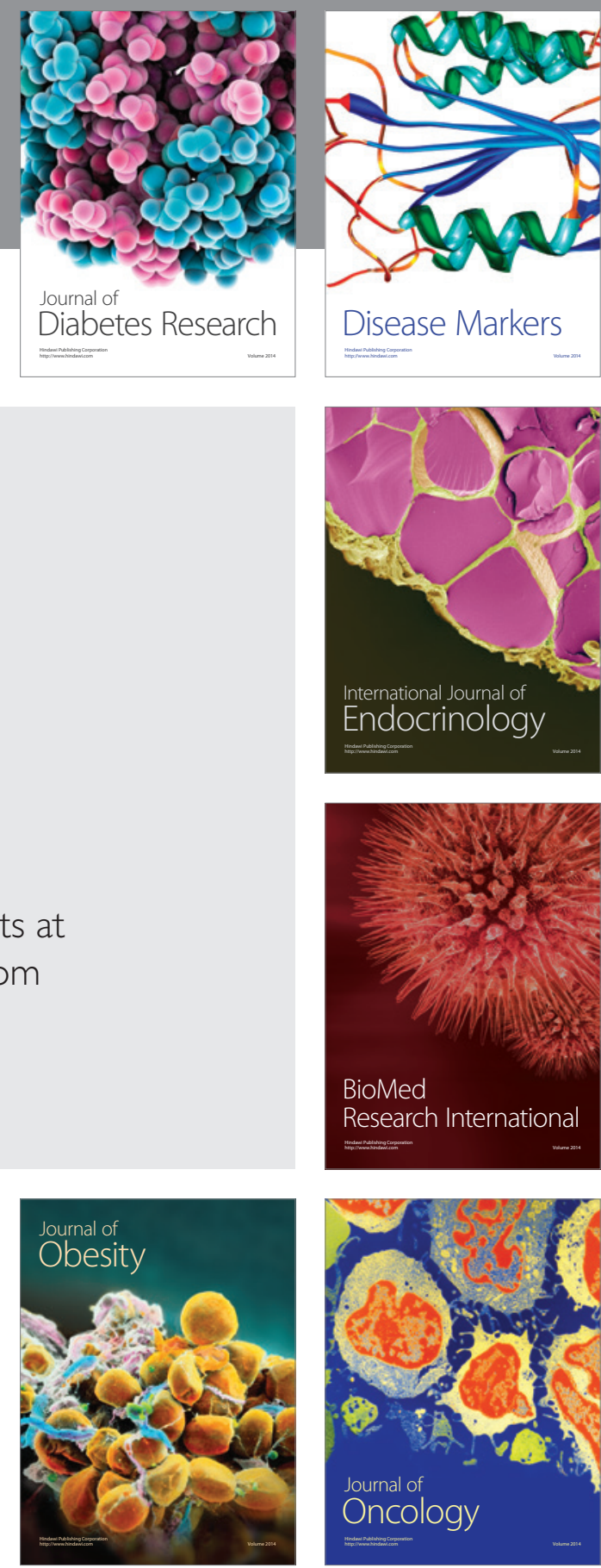

Disease Markers
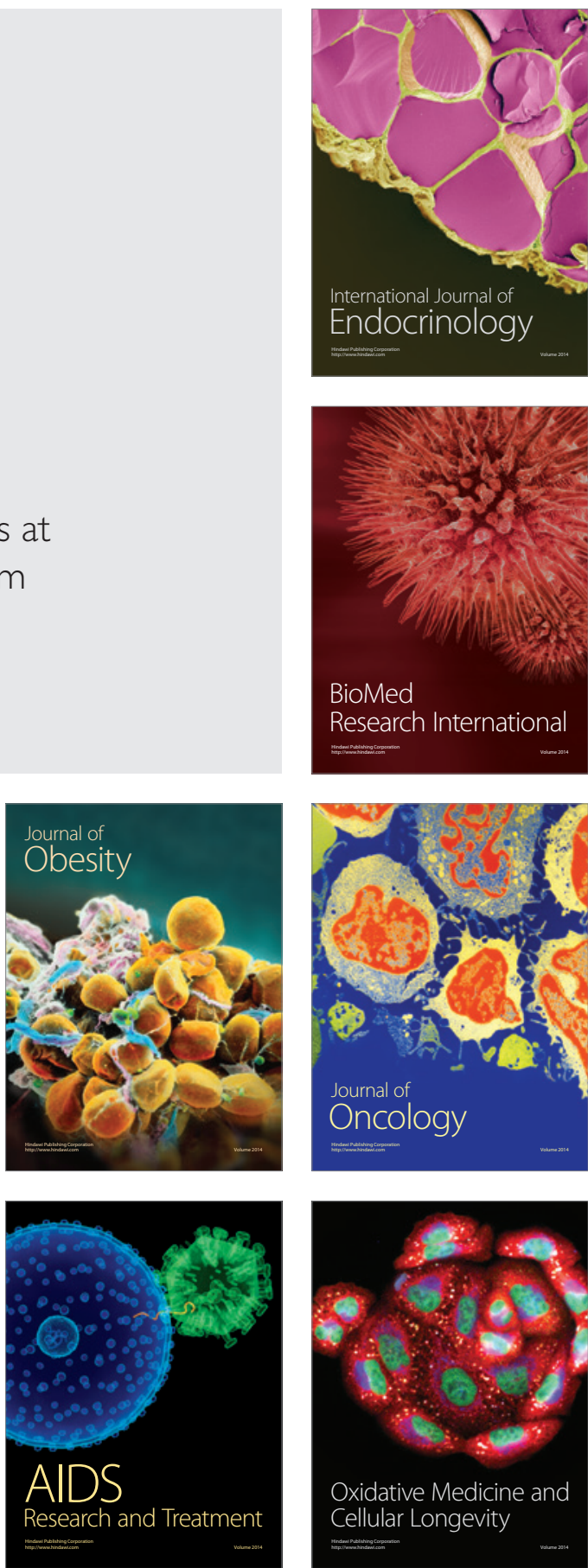